\title{
The Evolution of Clinical Audit as a Quality Improvement Tool in Gaza
}

\author{
Said Alyacoubi ${ }^{1}$, Loai Albarqouni ${ }^{2}$, Khamis Elessi ${ }^{1}$, and Bettina Bottcher ${ }^{1}$ \\ ${ }^{1}$ Islamic University of Gaza Faculty of Medicine \\ ${ }^{2}$ Bond University
}

April 27, 2020

\begin{abstract}
Rationale, aims and objective Clinical audit plays a fundamental role in improving the quality of patient care and hence, is considered a cornerstone of clinical governance. This study evaluates clinical audit as a newly introduced quality improvement tool in the healthcare system of the Gaza Strip. Methods: Medical students and healthcare professionals who completed audits between 2015 and 2018 were invited to fill in an online survey from October 12 to November 2, 2018. Data was collected on applied methods, supervision, training, re-auditing and subsequent improvements in practice. Results A total of 62 audits was collected. Former training in clinical governance was received by 55 authors (88.7\%) while a senior supervision was available in 56 audits (90.3\%). Audits were performed across different hospitals and specialties with 18 audits (29\%) in obstetrics, 16 audits $(25.8 \%)$ in medicine and 11 audits $(17.7 \%)$ each in surgery and paediatrics. A clear trend of increasing numbers of audits was observed with four audits (6.4\%) completed in 2015 and 24 audits (38.7\%) in 2018. Only 32 audits (51.6\%) were presented to the local staff. The audit cycle was only completed in 14 projects $(22.5 \%)$ with seven of them reporting improvements in practice. Conclusions A rise in the numbers of audits reflects a growing awareness of its key role in healthcare and patient safety. However, closure of loops and the actual implementation of recommendations are still lagging behind. Therefore, more focused efforts are needed to implement changes and ensure continuous evaluation of their effectiveness.
\end{abstract}

\section{The Evolution of Clinical Audit as a Quality Improvement Tool in Gaza}

Said Alyacoubi ${ }^{1 *}$, Loai Albaraqouni ${ }^{2},{\text { Khamis } \text { Elessi }^{1} \text {, Bettina Bottcher }}^{1}$

Corresponding author:

Dr Said Alyacoubi, MD MSc (Oxon)

Email address: said.alyacoubi@nhs.net

Telephone number: 07729907109

Institutional affiliations:

${ }^{1}$ Evidence-Based Medicine Unit, Faculty of Medicine, Islamic University of Gaza, Gaza, Palestine

${ }^{2}$ Centre for Research in Evidence-Based Practice, Bond University, Robina, Queensland, Australia

* Southampton University Hospitals NHS Foundation Trust, Southampton, UK

\section{Introduction}

Clinical audits play a fundamental role in improving the quality of clinical practice and patient safety 123 . The audit cycle involves observing practice, setting standards, comparing practice with standards, implementing changes and finally observing new practice ${ }^{4}{ }^{6}$. Closing the audit cycle is essential to achieve 
sustainable improvements in healthcare ${ }^{2} 78$. In practice, the cycle is often not completed, and the problems are left unresolved, which can potentially result in clinical auditing being a time- and resource-wasting activity 9101112 .

Clinical audit was implemented relatively recently as a quality improvement (QI) tool in the healthcare system of the Gaza Strip. For example, teaching concepts of QI work and clinical audit were only introduced into the curriculum of local medical schools in 2015. This led to the completion of a number of audit projects by medical students for the first time in $2015^{13} 1415$. A notable rise of QI work conducted by medical students was observed in the following years. In 2017, the Palestinian Ministry of Health (MoH) and the Palestinian Medical Council (PMC) introduced a general requirement for healthcare professionals, including interns and residents, to partake in QI activities. These measures sparked a wider engagement in audit activities as well as a broad awareness of the importance of QI work amongst medical students and healthcare professionals. However, evidence of monitoring of audit activities and subsequent improvements in clinical practice is still lacking. Consequently, concerns were raised regarding the effectiveness of clinical audits in improving the quality of patient in Gaza Hospitals.

Therefore, we decided to track and evaluate the clinical audits that were conducted in the Gaza Strip between 2015 and 2018. We also aimed to identify factors that would contribute towards producing more impactful audits in order to improve the quality of patient care in Gaza hospitals.

\section{Methods}

\section{Design and setting:}

An online survey was used to collect information on the audit projects completed in the Gaza Strip-Palestine between 2015 and 2018. The survey was disseminated through email communications and on local and institutional social media platforms; including those of the $\mathrm{MoH}$ and local universities. The survey was accessible from October 12 to November 2, 2018.

\section{Participants:}

$\mathbf{S}$ tudents, doctors and other healthcare professionals were invited to complete the survey if they had an audit project conducted during the specified period (2015-2018).

\section{Main outcome measures:}

Data was collected on different aspects of the audit process; including audit teams, purpose and location of study, applied methods, supervision, formal training, target population, sample size, data collection tools, outcomes, presentation, completion of cycle and subsequent improvement in practice.

\section{Results}

A total of 62 audits was registered via the survey. Students were involved in 46 audits (74.2\%) while practicing clinicians were only involved in 29 audits (46.7\%). Fifty audit groups (80.6\%) sought an approval from the General Directorate of Human Resources Development at the MoH, the body known to be responsible for approving studies, and/or the local healthcare facility administration before commencing their work. Training in clinical auditing was formerly received by 55 authors (88.7\%). Senior supervision was available in 56 audits (90.3\%), with one supervisor having been mentioned in 42 of them (67.7\%).

The majority of audits $(17 ; 27.4 \%)$ were multicentric; conducted at more than one healthcare facility. Thirteen audits $(20.9 \%)$ were conducted at one hospital (Al-Helal Al-Emirati Hospital for Obstetrics and Gynaecology). Audits were also performed in various specialties, including 18 audits (29\%) in obstetrics, 16 audits $(25.8 \%)$ in medicine, 11 audits $(17.7 \%)$ each in surgery and paediatrics, and six audits $(9.6 \%)$ in other specialties. A clear trend of increasing numbers of audits was observed with 4 audits (6.4\%) completed in 2015, 12 audits (19.3\%) in 2016, 22 audits (35.4\%) in 2017, and 24 audits (38.7\%) in 2018. Clear comparative standards were identified in 56 audits $(90.3 \%)$ while six audits $(9.7 \%)$ reported not setting standards at all. 
Among those with chosen standards, 40 audits (64.5\%) used international guidelines while only 14 audits $(22.5 \%)$ applied local practice guidelines (Table 1 ).

Improvement in documentation was recommended in 44 audits $(71 \%)$, development of national guidelines in 37 audits $(59.6 \%)$, staff training in 32 audits $(51.6 \%)$ and patient education in 14 audits $(22.5 \%) .32$ audits $(51.6 \%)$ were presented to the local staff at the healthcare facility where the work was originally conducted. 48 audits $(77.4 \%)$ were presented at other local meetings or conferences and 23 audits (37\%) were presented at national or international meetings. The results of five audits $(8 \%)$ were not presented anywhere and none of them had completed the cycle (Table 2 ).

The abstracts of 13 audits (20.9\%) were published in supplements of peer-reviewed journals. Finally, the audit cycle was completed in 13 projects (20.9\%), with only seven of them (11.3\%) reporting subsequent improvements in clinical practice (table 2). Of these seven audits, only one did not show improvements in practice following closing the loop.

\section{Discussion}

The lack of local and central registration systems makes it broadly difficult to track and evaluate the progress and development of QI work across the Gaza Strip. However, up until 2015, no evidence is available of any systematic QI work undertaken by medical students or healthcare practitioners in Gaza Hospitals.

The implementation of measures endorsing audit activities by local medical schools, MoH and PMC led to a growing awareness of the concepts of QI work and harnessed the cooperation between healthcare professionals and medical students to conduct clinical audits. This was reflected by a steady increase in the number of audits undertaken in local healthcare practice. An increasing number of abstract submissions to the international Lancet Palestinian Health Alliance (LPHA) annual conference was also observed between 2015 to 2018 with some positive impact reports ${ }^{16} 17$.

Crucially, the cycle has to be completed in order to assess the impact of the original audit. In this study, the cycle was found completed in only one fifth of all projects (20.9\%). This number is low but, nonetheless, similar to those reported in other studies ${ }^{9} 1819$ 20. Although such studies were undertaken in the context of a well-developed and supported audit programmes unlike that of this study, efforts should still be made towards achieving higher completion rates in our local practice and elsewhere.

Although the observed spike in audit numbers is encouraging, the impact of audit activities in Gaza is still lagging behind with only seven studies (11.3\%) reporting subsequent improvements in practice. In general, audits with local staff involvement and senior leadership proved to be most effective in initiating and leading improvements ${ }^{7} 89$. The provision of feedback to the local staff through presentation of results at internal meetings has also been shown to be effective ${ }^{12}$. Other measures such as the availability of dedicated support teams or the provision of protected time for QI work ${ }^{5} 21222324$ are often difficult to implement in the case of low-income countries ${ }^{2526}$. Therefore, it is imperative to focus on factors that can potentially be supported in such settings in order to produce QI work with the highest possible impact ${ }^{2726}$.

\section{Conclusions}

While only few audits were conducted prior to 2015, the constant rise in numbers proves a growing awareness of the benefits associated with QI efforts. However, the actual implementation of changes is still lagging behind. Hence, focused efforts supported by both clinical and administrative leaderships are needed to implement recommendations and action plans. A limitation of this work is the number of audits missing from our study.

\section{Funding}

This study received no funding.

\section{Conflict of interest statement:}

The authors have no conflict of interest to declare. 


\section{References}

1. Principles for Best Practice in Clinical Audit. Int J Health Care Qual Assur. 2002;15(7):ijhcqa.2002.06215gae.004. doi:10.1108/ijhcqa.2002.06215gae.004

2. Gnanalingham J, Gnanalingham MG, Gnanalingham KK. An Audit of Audits: Are we Completing the Cycle? J R Soc Med. 2001;94(6):288-289. doi:10.1177/014107680109400609

3. Øvretveit J. Understanding the conditions for improvement: Research to discover which context influences affect improvement success. BMJ Qual Saf. 2011. doi:10.1136/bmjqs.2010.045955

4. Pawson R, Greenhalgh T, Harvey G, Walshe K. Realist review - A new method of systematic review designed for complex policy interventions. J Heal Serv Res Policy. 2005. doi:10.1258/1355819054308530

5. Kaplan HC, Froehle CM, Cassedy A, Provost LP, Margolis PA. An exploratory analysis of the Model for Understanding Success in Quality. Health Care Manage Rev. 2013. doi:10.1097/HMR.0b013e3182689772

6. Spurgeon P, Mazelan PM, Barwell F. Medical engagement: A crucial underpinning to organizational performance. Heal Serv Manag Res. 2011. doi:10.1258/hsmr.2011.011006

7. McDonald KM, Schultz EM, Chang C. Evaluating the state of quality-improvement science through evidence synthesis: insights from the closing the quality gap series. Perm J. 2013. doi:10.7812/TPP/13-010

8. Ivers NM, Sales A, Colquhoun H, et al. No more "business as usual" with audit and feedback interventions: Towards an agenda for a reinvigorated intervention. Implement Sci. 2014. doi:10.1186/1748-5908-9-14

9. Taylor L, Jones S. Clinical governance in practice: Closing the loop with integrated audit systems. J Psychiatr Ment Health Nurs. 2006. doi:10.1111/j.1365-2850.2006.00945.x

10. Iqbal H., Pidikiti P. Audit of Orthopaedic Audits in an English Teaching Hospital: Are We Closing the Loop? Open Orthop J. 2010. doi:10.2174/1874325001004010188

11. Wagner DJ, Durbin J, Barnsley J, Ivers NM. Beyond quality improvement: Exploring why primary care teams engage in a voluntary audit and feedback program. BMC Health Serv Res. 2017. doi:10.1186/s12913017-2765-3

12. Ivers N, Jamtvedt G, Flottorp S, et al. Audit and feedback: Effects on professional practice and healthcare outcomes. Cochrane Database Syst Rev. 2012. doi:10.1002/14651858.CD000259.pub3

13. Alyacoubi S, Abuowda Y, Albarqouni L, Böttcher B, Elessi K. Inpatient management of communityacquired pneumonia at the European Gaza Hospital: a clinical audit. Lancet (London, England). 2018;391. doi:10.1016/S0140-6736(18)30406-9

14. Abukhalil M, Bottcher B, Mehjez O, Alankah L, Abuyusuf M, Hasan S. Medical records of emergency caesarean sections in the Gaza Strip: a clinical audit. Lancet. 2018. doi:10.1016/s0140-6736(18)30351-9

15. Musallam R, Salem N, Al Halol R, Al Deeb H, Bottcher B, AlHamaida H. Management of pregnancy loss in the first trimester: a retrospective audit. Lancet (London, England). 2018. doi:10.1016/S01406736(18)30400-8

16. Alrumi N, Aghaalkurdi M, Habib H, Abed S, Böttcher B. Infection control measures in neonatal units: implementation of change in the Gaza-Strip. J Matern Neonatal Med. 2019. doi:10.1080/14767058.2019.1576168

17. Zimmo KM, Laine K, Fosse E, et al. Impact of animated instruction on tablets and hands-on training in applying bimanual perineal support on episiotomy rates: an intervention study. Int Urogynecol J. 2018. doi:10.1007/s00192-018-3711-6

18. Gnanalingham J, Gnanalingham MG GK. An Audit of Audits: Are we Completing the Cycle? J R Soc Med. 2001.

19. John CM. An audit of paediatric audits. Arch Dis Child. 2004;89(12):1128-1129. doi:10.1136/adc.2003.032565 
20. Guryel E, Acton K, Patel S. Auditing orthopaedic audit. Ann R Coll Surg Engl. 2008. doi:10.1308/003588408X318147

21. Johnston G, Crombie IK, Davies HTO, Alder EM, Millard A. Reviewing audit: Barriers and facilitating factors for effective clinical audit. Qual Heal Care. 2000. doi:10.1136/qhc.9.1.23

22. Anderson P, Fee P, Shulman R, Bellingan GJ, Howell DCJ. Audit of audit: Review of a clinical audit programme in a teaching hospital intensive care unit. Br J Hosp Med. 2012. doi:10.12968/hmed.2012.73.9.526

23. Coles E, Wells M, Maxwell M, et al. The influence of contextual factors on healthcare quality improvement initiatives: What works, for whom and in what setting? Protocol for a realist review. Syst Rev. 2017. doi:10.1186/s13643-017-0566-8

24. Kaplan HC, Brady PW, Dritz MC, et al. The influence of context on quality improvement success in health care: A systematic review of the literature. Milbank Q. 2010. doi:10.1111/j.1468-0009.2010.00611.x

25. Hut-Mossel L, Welker G, Ahaus K, Gans R. Understanding how and why audits work: Protocol for a realist review of audit programmes to improve hospital care. BMJ Open. 2017. doi:10.1136/bmjopen-2016015121

26. Pirkle CM, Dumont A, Zunzunegui MV. Criterion-based clinical audit to assess quality of obstetrical care in low- and middle-income countries: A systematic review. Int J Qual Heal Care. 2011. doi:10.1093/intqhc/mzr033

27. Bottcher B, Abu-El-Noor N, Abuowda Y, et al. Attitudes of doctors and nurses to patient safety and errors in medical practice in the Gaza-Strip: a cross-sectional study. BMJ Open. 2019;9(8):e026788. doi:10.1136/bmjopen-2018-026788

\section{Hosted file}

Tables.doc available at https://authorea.com/users/315846/articles/446073-the-evolution-of-clinicalaudit-as-a-quality-improvement-tool-in-gaza 